

BJRS
BRAZILIAN JOURNAL

$\mathrm{OF}$

RADIATION SCIENCES

07-2A (2019) 01-07

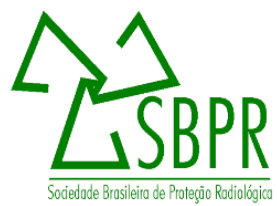

\title{
CAX and Xsel: a software bundle to aid in automating NAA spectrum analysis
}

\author{
G. S. Zahn; L. S. Junqueira; F. A. Genezini \\ Guilherme S. Zahn, Iberê Ribeiro Filho, Frederico A. Genezini \\ Instituto de Pesquisas Energéticas e Nucleares (IPEN / CNEN - SP, Av. Professor Lineu Prestes 224 \\ 05508-000 São Paulo,SP-gzahn@ipen.br
}

\begin{abstract}
In this work, the scripting capabilities of Genie-2000 were used to develop a software (CAX) that automatically analyses all spectrum files in either ORTEC ${ }^{\text {TM's }}$ CHN or CANBERRA's MCA or CNF formats in a folder, generating two output files: a print-ready text file (.DAT) and a Comma-Separated Values (.CSV) file which can be easily imported in any major spreadsheet software. A second tool, named Xsel, consists in an EXCEL ${ }^{\mathrm{TM}}$ spreadsheet which automatically identifies the spectrum lines associated with the elements of interest, generating a clean output sheet which can be easily imported in a general spreadsheet that calculates the concentrations; an important addition to this spreadsheet is the ability to automatically recalibrate each spectrum, increasing the reliability of the peak identification procedure. These software tools are already used in daily routines at IPEN's Neutron Activation Laboratory, greatly reducing the time required for sample analyses, as well as reducing the possibility of transcription errors.
\end{abstract}

Keywords: NAA, automated analysis. 


\section{INTRODUCTION}

The analysis of gamma-ray spectra is a key part of any application involving gamma-ray spectrometry, and there is plenty of software to choose from to perform this task [1]. These software tools usually perform a set of functions: read the spectrum file; import or adjust the energy -- and, sometimes, FWHM (Full Width at Half Maximum, a measure of the system's energy resolution) -- calibration; locate the gamma-ray peaks in the spectrum; determine the precise location, width and area of each peak; output the results.

While seemingly simple, many of these tasks differ strongly from software to software. Some software, for instance, can only import spectra in a very narrow selection of formats -- if the software also controls the data acquisition, it may import only its own native format. Also, most acquisition software can only export spectra in its native format, and that changes from time to time, even for the same manufacturer. Thus, in a laboratory that has equipment from more than one manufacturer, and of distinct age, it is not uncommon to have to deal daily with three or four distinct spectrum formats, increasing the importance of using a single platform that can import spectra in many different formats.

Also, the results from these fits will most likely be used as input for further data analysis, so it is important that the output can be easily integrated in the next steps of the analysis. In this point, also, software may differ greatly, either outputting the results directly to a printer (usual in the most ancient software), to a regular output file, or even be very customizable, accepting custom report formats.

Finally, regarding the spectrum analysis process, software may also differ greatly. Some may offer a possibility to perform energy and FWHM calibration, other may only import the calibrations from the spectrum file, and HypermetPC even performs its FWHM calibration "on the fly" [2]. The quality of the peak-finding and fitting routines is also very variable, as shown by the authors in a previous study [1].

This way, in order to simplify and unify the spectrum analysis process in a complex laboratory with several different detectors and diverse possibilities of data analyses, it is essential to choose a software that can both read spectra in different formats and output the results in an easy-to-use format. 
Once the spectrum analyzed, the association of peaks in the output list to the specific elements to be analyzed in a NAA (Neutron Activation Analysis) experiment, although seemingly a simple task, requires a great deal of care as the precise calibration of spectra slightly change from one measurement to another. Also, it is common to find two or three peaks very close to each other, and selecting the correct one usually requires caution and experience.

\section{SOFTWARE DESIGN}

In order to develop the present software, two main choices were required: that of a suitable peak analysis software, and that of a framework in which to develop the peak association tool.

For analyzing spectrum files, CANBERRA's Genie-2000 [3] was chosen, because of its superior performance in the peak fitting task [1] as well as for its versatility, as it is able to import spectrum data in several distinct formats and offers great scripting capabilities. In fact, every action available in Genie-2000's Graphical User Interface can also be performed by means of a command-line tool, allowing for an easy, seamless integration of some third-party software.

For the peak association task, the choice was to develop it using the ubiquitous EXCEL ${ }^{\mathrm{TM}}$ spreadsheet software, as it has built-in all the tools required both for the selection of peaks and recalibration. Moreover, the use of a graphical tool already known by virtually all users in the lab eases much of the tasks of properly formatting the output and transferring it to a proper calculus spreadsheet.

\subsection{CAX}

The CAX ("Convert and Analyze for eXcel") software was written in the Pascal language and compiled using the open-source FreePascal compiler [4]. Although FreePascal is available for many platforms, so that CAX might be ported to all of those, the software makes use of many of Genie2000's executables, which run only under 32-bit Windows -- another very important limitation is that it will only run in systems with a working registered version of Genie-2000. 
At this time, CAX will run exclusively in command-line mode, fitting all the spectra found in the current folder using the same settings. The syntax is:

\section{CAX [/N] [/L] [Calibration File]}

where exactly one of the parameters must be used. "/N" tells CAX to use the internal calibrations found in the spectrum file itself (i.e., no recalibration whatsoever); "/L" lists all calibration files found in the calibrations folder (at present this is not configurable, and all calibrations are expected to be found at the $c: \backslash a a n \backslash c a \Lambda$ folder); and "Calibration File" is the name of the calibration file to use.

The command will result in the creation of two ASCII report files for each spectrum file in the folder, a printable one with the ".RPT" extension and an EXCEL ${ }^{\mathrm{TM}}$-compatible one with the ".CSV" extension. Also, if the original spectrum was not in the .CNF format, a CNF file will be created.

In short, the software execution sequence is as follows:

1. Locates all spectrum files within the current folder, using Pascal's FindFirst function; if report files are found, automatically creates a backup folder with the current date and time as name, to avoid overwriting previous backups, and moves the reports to that folder;

2. Runs filecnvt, a tool from Genie-2000's which imports the spectrum file into its native .CNF format;

3. If requested, imports calibration from a saved file, using Genie-2000's movedata, with the lecal, parameter, which means that: a) if there is a corresponding section in the spectrum file, it will be overwritten; and $b$ ) that the section to be copied is the energy calibration (which includes FWHM calibration, too) - the energy calibration files are .CNF files with proper energy calibration, one per spectrometer, stored in a specific sub-folder of the CAX folder (at the present time, rigorously $c: \backslash a a n \backslash c a l$ );

4. Runs Genie-2000's analyze command - this tool performs the peak finding and fitting, and also creates a report; this requires a specific "Analysis Sequence" script with the .ASF extension, created inside the main Genie-2000 GUI, which contains the steps to be run at the analysis stage and their options -- in this case, peak finding, with the proper settings, and peak fitting, also with a set of parameters; these settings and parameters may depend on the laboratory's specific demands, but using Genie's default options is generally a good starting point. 
5. As the regular ".RPT" Genie-2000 report uses international number conventions (with dots as decimal separators), incompatible with most spreadsheet software in Brazil, CAX then runs its own simple external file converter (RPT2CSVBR, also written in Pascal), which reads the report file, replacing dots with commas and blank spaces with semicolons (with an additional step to make sure that sequential spaces are treated as a single one), so that the output file conforms to the Brazilian CSV (Comma Separated Values) standard, where commas are decimal separators and semicolons are used to separate fields.

\subsection{Xsel}

This tool consists in an EXCEL ${ }^{\mathrm{TM}}$ spreadsheet which reads the output from CAX and produces a cleaner output, with only the lines associated with peaks from the nuclides of interest. It consists in a spreadsheet with four tabs:

- "Elements", with a list with all energy peaks of interest and the related elements (may be slightly different for each user);

- "Input", where the full output from CAX, which contains the actual data, must be pasted;

- "Calc", where all the calculations are actually performed and;

- "Output", where it outputs the list of selected peaks with their corrected energy, count rate and uncertainty - this tab, too, may require some easy to perform adaptations to adequate to the user's specific calculus spreadsheet input format.

The sequence performed by Xsel is as follows:

1. To take into account possible calibration variations, it first defines a rough energy-dependent tolerance $(\mathrm{T})$ for each of the energies in the "Elements" tab as:

2. $T=0.5+0.0015 \times E \gamma$

3. where $E \gamma$ is the expected peak energy; this way, an experimental peak of energy $E$ is associated with the transition $E \gamma$ if $(E \gamma-T)<E<(E \gamma+T)$;

4. For each energy in the list of expected transitions, it finds the first line in the experimental peak list with an energy greater than $E \gamma$-T using EXCEL ${ }^{\mathrm{TM}}$ 's functions CORRESP (in English, MATCH) and copies it to the new list using the function DESLOC (in English, OFFSET);

5. To check if the peak is in the expected interval, it verifies that the experimental energy value is below $E \gamma+T-$ if this is not true, the cell is left blank; 
6. To correct for the possible calibration deviations, the spreadsheet then performs a quick recalibration of the experimental energies using EXCEL ${ }^{\mathrm{TM}}$ 's built-in PREVISÂO function (FORECAST, in English) which performs a linear fit of the experimental energies versus the expected values - assuming that most peaks were correctly identified, their new energy values should be much closer to the expected ones and a much tighter tolerance can be defined;

7. Using the corrected energies, the CORRESP and DESLOC functions are used again, now with a smaller tolerance, to precisely identify and copy the correct values to the output list - our tests found that $\mathrm{T}=0.5 \mathrm{keV}$ was an optimal tolerance value for a detector with $2 \mathrm{keV}$ resolution for the $1332 \mathrm{keV}$ gamma ray peak of ${ }^{60} \mathrm{Co}$.

\section{RESULTS AND DISCUSSION}

In the daily use, $C A X$ was found to be very useful and time-efficient (it takes approximately $5 \mathrm{~s}$ to analyze each spectrum file, while the manual analysis takes at least 10 times longer), although the command-line interface was found to be somewhat counterintuitive for most users. The other complaint from some users was related to the process of importing the results in their own calculus spreadsheets, and solving that was the aim of developing Xsel.

Xsel proved to be very intuitive for most users, and it actually proved to be very effective in separating the correct peak from a list of close energy values - in fact, an accidental but very interesting finding was that the use of Xsel helped determining peaks heavily distorted by some spectral interference, as in this case the peak energy usually falls slightly, but surely, outside the strict tolerance range defined.

\section{CONCLUSION}

Although it runs only in command-mode, in computers where Genie-2000 is properly installed, the CAX software is already being used in the daily routine of many users in IPEN's Neutron Activation Laboratory. The main advantages of its use are the increased analysis speed and the ease with which the reports can be imported in spreadsheets for the calculation of concentrations, avoiding the need to retype the results of the peak analysis manually; as a bonus, the software has also served as 
an incentive for users to keep their spectrum files, which previously were frequently discarded after fitting.

As for its counterpart Xsel, its use is still growing, but the ability to identify and eliminate peaks that were distorted by some very close spectral interference proved to be a welcome tool for new users.

\section{REFERENCES}

1. ZAHN, G. S., GENEZINI, F. A., MORALLES, M. Evaluation Of Peak-Fitting Software For Gamma Spectrum Analysis, arXiv:1511.04362, 2015.

2. FAZEKAS, B., et al., Introducing HYPERMET-PC for automatic analysis of complex gammaray spectra, Journ. Radioanal. Nucl. Chem., v. 215, p.271-277, 1997.

3. CANBERRA INDUSTRIES INC., Genie-2000 Spectroscopy System -- operations manual, Meriden, USA, 2006.

4. FREE PASCAL DEVELOPMENT TEAM Free Pascal -- Open source compiler for Pascal and Object Pascal, Available at: https://www.freepascal.org/. Last Acessed in 16-November-2017. 